University of Wisconsin - Madison

$\mathrm{MAD} / \mathrm{PH} / 827$

KA TTP94-2

March 1994

\title{
JET PRODUCTION IN POLARIZED LEPTON-HADRON SCATTERING
}

\author{
E. Mirkes ${ }^{a}$ and C. Ziegler ${ }^{b}$ \\ ${ }^{a}$ Physics Department, University of Wisconsin, Madison WI 53706, USA \\ ${ }^{b}$ Institut für Theoretische Teilchenphysik, Universität Karlsruhe, Kaiserstr. 12, \\ 75128 Karlsruhe 1, Germany
}

\begin{abstract}
We calculate exact analytical expressions for $\mathcal{O}\left(\alpha_{s}\right) 3$-jet and $\mathcal{O}\left(\alpha_{s}^{2}\right)$ 4 -jet cross sections in polarized deep inelastic lepton nucleon scattering. Introducing an invariant jet definition scheme, we present differential distributions of 3 - and 4-jet cross sections in the basic kinematical variables $x$ and $W^{2}$ as well as total jet cross sections and show their dependence on the chosen spin-dependent (polarized) parton distributions. Noticebly differences in the predictions are found for the two extreme choices, i.e. a large negative sea-quark density or a large positive gluon density. Therefore, it may be possible to discriminate between different parametrizations of polarized parton densities, and hence between the different physical pictures of the proton spin underlying these parametrizations.
\end{abstract}





\section{INTRODUCTION}

The spin structure of the longitudinally polarized proton has attracted much theoretical interest during the past years. This is mainly due to the disagreement between the theoretical prediction of the Ellis-Jaffe sum rule [1] for the first moment of the polarized structure function $g_{1}\left(x, Q^{2}\right)$ and the combined SLACEMC-data [2]. The experimental results which turn out to be much smaller than expected suggest two extreme scenarios: a large negative polarization for the sea-quarks [3] or alternatively a large positive polarization of gluons in the longitudinally polarized proton [4.5] (see also the discussion in sect. II). A combination of both is also possible.

There have been many suggestions for measuring polarized parton distributions with hard processes [6]. Only processes where the polarized gluon distribution $\triangle g$ enters at leading order are a promising source for measuring the polarized parton distributions.

In this paper we study 3 - and 4-jet cross sections in polarized deep inelastic lepton-nucleon processes using recently proposed polarized quark and gluon distributions, $\triangle q$ and $\triangle g$ respectively.

To $\mathcal{O}\left(\alpha_{s}\right)$ one has 3 -jet final states*. The relevant subprocesses are $\gamma^{*} q \longrightarrow$ $G q$ and $\gamma^{*} G \longrightarrow q \bar{q}$. In $\mathcal{O}\left(\alpha_{s}^{2}\right)$ the following subprocesses contribute to the 4jet production: $\gamma^{*} q \longrightarrow G G q, \gamma^{*} q \longrightarrow q q \bar{q}$ and $\gamma^{*} G \longrightarrow q \bar{q} G$. All partons are assumed to be massless. We present exact analytical expressions for the relevant 3- and 4-jet partonic hard scattering cross sections, using polarized beams and polarized targets, and specify how these partonic cross sections are folded with the respective polarized parton densities to obtain the DIS hadronic cross section. Introducing an invariant jet definition scheme we present differential distributions of 3- and 4-jet rates.

Large differences in the differential distributions are found for the two extreme choises for the polarized parton distributions made in the literature i.e. a large negative sea-quark density or a large positive gluon density. Thus, the analysis of jet production in polarized deep inelastic scattering allows to extract $\Delta \bar{q}$ and/or $\Delta G$ from future measurements and it may be possible to discriminate between the distinctly different physical pictures of the proton spin underlying these parametrizations.

The paper is organized as follows: In section II we introduce our notations and some useful formulae. In section III we discuss the problem of defining jets in deep inelastic scattering and present analytical results for the $O\left(\alpha_{s}\right) 3$-jet cross

\footnotetext{
*We include the target jet when counting the number of produced jets.
} 
sections. Section IV contains analytical formulae for the $O\left(\alpha_{s}^{2}\right) 4$-jet cross sections. We specify in detail how the partonic hard scattering cross sections are folded with the respective parton distribution functions. In setion $\mathrm{V}$ the set of polarized parton distribution functions used in our calculations are reviewed. Section VI contains our numerical results. We discuss overall kinematical cuts that we choose to define our deep inelastic scattering phase space. Further cuts are then imposed on the invariant jet-jet masses $s_{i j}$ to define the resolved 3- and 4-jet phase space. We present predictions for the 3 - and 4-jet cross sections and show distributions in the kinematical variables $x$ and $W^{2}$. Section VII contains our summary. Finally, detailed analytical formulae for the 4-jet partonic matrix elements are relegated to the appendix.

\section{KINEMATICS OF POLARIZED DIS AND THE STRUCTURE FUNCTION $G_{1}$}

Consider deep inelastic lepton-proton scattering ( $X$ is an arbitrary hadronic final state)

$$
l_{1}(l)+H(P) \longrightarrow l_{2}\left(l^{\prime}\right)+X\left(P_{f}\right)
$$

The particle momenta are given within the brackets.

Reaction (11) proceeds via the exchange of an intermediate vector boson $V=$ $\gamma^{*}, Z, W^{ \pm}$. In this paper we will only consider the exchange by a virtual photon. We denote the $\gamma^{*}$-momentum by $q$, the absolute square by $Q^{2}$, the cms energy by $\mathrm{s}$, the square of the final hadronic mass by $W^{2}$ and introduce the scaling variables $x$ and $y$ :

$$
\begin{aligned}
q & =l-l^{\prime} \\
Q^{2} & \equiv-q^{2}=x y s>0 \\
s & =(P+l)^{2} \\
W^{2} & \equiv P_{f}^{2}=(P+q)^{2} \\
x & =\frac{Q^{2}}{2 P q} \quad(0<x \leq 1) \\
y & =\frac{P q}{P l} \quad(0<y \leq 1) .
\end{aligned}
$$

For fixed s, only two variables in (2) are independent, since e. g.

$$
x W^{2}=(1-x) Q^{2}, \quad Q^{2}=x y s .
$$

For polarized lepton and polarized target scattering, the inclusive (with respect to the outgoing hadrons) cross section for process (四) can be written as 


$$
\frac{d \Delta \sigma}{d x d y} \equiv \frac{d^{2} \sigma^{\uparrow \uparrow}}{d x d y}-\frac{d^{2} \sigma^{\uparrow \downarrow}}{d x d y}=\frac{8 \pi \alpha^{2}}{q^{2}}\left[\left\{2-y-\frac{M x y}{E}\right\} g_{1}\left(x, Q^{2}\right)-\frac{2 M x}{E} g_{2}\left(x, Q^{2}\right)\right]
$$

The left arrow above $\sigma$ denotes the polarization of the incoming lepton with respect to the direction of its momentum. The right arrow stands for the polarization of the proton parallel or anti-parallel to the polarization of the incoming lepton. $g_{1}\left(x, Q^{2}\right)$ and $g_{2}\left(x, Q^{2}\right)$ are the polarized structure functions for longitudinal and transverse spin respectively. At large energies, only $g_{1}$ contributes.

In the QCD improved parton model, the polarized structure function $g_{1}^{P}$ of the proton can be expressed in the lowest-order approach byt

$$
2 g_{1}^{P}\left(x, Q^{2}\right)=\frac{4}{9} \Delta u_{v}\left(x, Q^{2}\right)+\frac{1}{9} \Delta d_{v}\left(x, Q^{2}\right)+\frac{4}{3} \Delta \bar{q}\left(x, Q^{2}\right)
$$

We have taken an $\mathrm{SU}(3)$-flavour symmetric polarized sea $\Delta \bar{q} \equiv \Delta \bar{u}=\Delta \bar{d}=\Delta \bar{s}=$ $\Delta s$ where $\Delta u_{v}$ and $\Delta d_{v}$ are the polarized valence quark distributions and

$$
\Delta q\left(x, Q^{2}\right)=q_{+}\left(x, Q^{2}\right)-q_{-}\left(x, Q^{2}\right) .
$$

In (5) $q_{+}\left(q_{-}\right)$denotes a parton of flavour $q$ in the proton whose spin is aligned (anti-aligned) to the proton's spin.

Experimental measurements of the asymmetry

$$
A_{1}^{P}\left(x, Q^{2}\right) \equiv \frac{2 x g_{1}^{P}\left(x, Q^{2}\right)}{F_{2}^{P}\left(x, Q^{2}\right)}
$$

provide us with a determination of $g_{1}^{P}\left(x, Q^{2}\right)$. In (6) $F_{2}^{P}\left(x, Q^{2}\right)$ is the unpolarized structure function, i.e. $F_{2}^{P}\left(x, Q^{2}\right)=x \sum_{q} e_{q}^{2}\left[q\left(x, Q^{2}\right)+\bar{q}\left(x, Q^{2}\right)\right]$.

Writing $g_{1}^{P}\left(Q^{2}\right) \equiv \int_{0}^{1} g_{1}^{P}\left(x, Q^{2}\right) d x$ as

$$
g_{1}^{P}\left(Q^{2}\right)=\frac{1}{2}\left[\frac{4}{9} \Delta u\left(Q^{2}\right)+\frac{1}{9} \Delta d\left(Q^{2}\right)+\frac{1}{9} \Delta s\left(Q^{2}\right)\right]
$$

and using the $F / D$ ratio of the hyperon decay and the Bjorken sum rule, the following values for the first moments of the polarized quark densities have been obtained [2,8,9]

$$
\begin{aligned}
& \Delta u=0.78 \pm 0.06 \\
& \Delta d=-0.47 \pm 0.06 \\
& \Delta s=-0.19 \pm 0.06
\end{aligned}
$$

\footnotetext{
${ }^{\dagger}$ We follow the notation of Glück, Reya, Vogelsang []].
} 
and

$$
\Delta q \equiv \Delta u+\Delta d+\Delta s=0.12 \pm 0.17
$$

Therefore the total spin carried by the quarks and antiquarks in a polarized proton is small and actually compatible with zero. This is the famous EMC result. Recently, new data from the SMC muon-deuteron scattering experiment [10] and from the SLAC muon-He scattering experiment [11] are available. It is shown in [12], that a global average of the present existing date yields $\Delta q \equiv \Delta u+\Delta d+\Delta s=$ $0.22 \pm 0.10$ which is still not very different from the value in (5).

Several groups have suggested that the difference between the Ellis-Jaffe sum rule [1] (the sea contribution was set to zero in the Ellis Jaffe sum rule) and the EMC result could be resolved by a larger higher correction due to a large gluon polarization [4,5] or by a large sea contribution [3]. Various sets of helicity difference distribution functions have been developed based on these ideas. An independent determination of the gluon spin densities are therefore crucial in the understanding of the spin structure of the proton.

In the following, we study $\mathcal{O}\left(\alpha_{s}\right)$ 3-jet and $\mathcal{O}\left(\alpha_{s}^{2}\right)$ 4-jet production in polarized DIS (using the polarized parton distributions proposed in [13]) and show that these processes yield strong information on the spin-dependent quark and gluon densities.

\section{JET CROSS SECTIONS IN $\mathcal{O}\left(\alpha_{s}\right)$}

The Quark-Parton model $\left(\mathcal{O}\left(\alpha_{s}^{0}\right)\right)$ process

$$
l_{1}(l)+q(p=\eta P) \longrightarrow l_{2}\left(l^{\prime}\right)+q\left(p_{1}\right)
$$

predicts two and only two jets in the final state, namely the struck quark jet and the hadron remnant jet which leads to the prediction of two back-to-back jets in the hadronic center-of-mass-frame.

The $\mathcal{O}\left(\alpha_{s}\right)$ tree graph processes (see fig. 1a,b for the relevant boson-parton subprocesses)

$$
\begin{gathered}
l_{1}(l)+\stackrel{(-)}{q}(p) \longrightarrow l_{2}\left(l^{\prime}\right)+\stackrel{(-)}{q}\left(p_{1}\right)+G\left(p_{2}\right) \\
l_{1}(l)+G(p) \longrightarrow l_{2}\left(l^{\prime}\right)+q\left(p_{1}\right)+\bar{q}\left(p_{2}\right)
\end{gathered}
$$

give rise to 3 -jet production. There are singular regions in the phase space of the $\mathcal{O}\left(\alpha_{s}\right)$ tree graph cross sections. The singular behaviour corresponds to the situation where two (or more) partons are irresolvable and have to be counted as contributions to the 2-jet cross section. Parts of these singular cross section contributions will cancel against corresponding singular terms of the $\mathcal{O}\left(\alpha_{s}\right)$ virtual 
2-parton corrections. A remaining initial state mass singularity has to be absorbed into the polarized parton densities.

In order to calculate a 3 -jet cross section we have to define what we call 3 jets by introducing a resolution criterion. As has been elaborated in detail in 14, 15 energy-angle cuts are not suitable for an asymmetric machine with its strong boosts from the hadronic cms to the laboratory frame. As a jet resolution criterion we use the invariant mass cut criterion introduced in [14 such that

$s_{i j} \geq M_{c}^{2}=\max \left\{y_{c u t} W^{2}, M_{0}^{2}\right\}=\max \left\{y_{c u t}\left(p_{1}+p_{2}+p_{3}\right)^{2}, M_{0}^{2}\right\} \quad(i, j=1,2,3 ; i \neq j)$

where $y_{c u t}$ is the resolution parameter and $s_{i j}$ is the invariant mass of any two final state partons, including the remnant jet with momentum $p_{3}=(1-\eta) P$. $M_{0}$ is a fixed mass cut which we have introduced in order to clearly separate the perturbative and non-perturbative regime in the case where $W^{2}$ is small (see the detailed discussion in sect. VI). The 3-jet phase space is represented by the kinematical variables

$$
x_{p}=\frac{Q^{2}}{2 p q}=\frac{x}{\eta} \quad z=\frac{p p_{1}}{p q} \quad \text { and } \quad \phi .
$$

The angle $\phi$ denotes the angle between the parton plane $\left(\vec{p}, \vec{p}_{1}\right)$ and the lepton plane $\left(\vec{l}_{1}, \vec{l}_{2}\right)$ in the $\vec{p}+\vec{q}=0$ center of mass frame.

The $\mathcal{O}\left(\alpha_{s}\right) 3$-jet cross section for polarized DIS is then obtained by

$$
\begin{aligned}
\frac{d^{2} \Delta \sigma}{d x d y}= & \frac{2 \pi \alpha^{2}(2-y)}{Q^{2}} \frac{\alpha_{s}\left(\mu_{R}^{2}\right)}{2 \pi} \int_{\eta_{\min }}^{\eta_{\max }} \frac{d \eta}{\eta} \int_{z_{\min }}^{z_{\max }} d z \int_{0}^{2 \pi} \frac{d \phi}{2 \pi} \\
& \left\{I_{\Delta q}^{(0)}+I_{\Delta G}^{(0)}+\frac{\sqrt{1-y}}{2-y} \cos \phi\left(I_{\Delta q}^{(1)}+I_{\Delta G}^{(1)}\right)\right\} .
\end{aligned}
$$

The integration limits in $\eta$ and $z$ originate from our jet resolution criterion (11) such that

$$
\begin{aligned}
& z_{\min }=\frac{M_{c}^{2}}{y s(1-\eta)}=1-z_{\max }, \\
& \eta_{\min }=x+\frac{M_{c}^{2}}{y s}, \quad \eta_{\max }=1-\frac{2 M_{c}^{2}}{y s} .
\end{aligned}
$$

The 3 -jet integrands are given by $(i=0,1)$

$$
\begin{aligned}
I_{\Delta q}^{(i)} & =\sum_{f=1}^{n_{f}} e_{f}^{2}\left(\Delta q\left(\eta, \mu_{F}^{2}\right)+\Delta \bar{q}\left(\eta, \mu_{F}^{2}\right)\right) \Delta\left|\mathcal{M}^{(i)}\right|_{q \rightarrow q G}^{2}, \\
I_{\Delta G}^{(i)} & =\left(\sum_{f=1}^{n_{f}} e_{f}^{2}\right) \Delta G\left(\eta, \mu_{F}^{2}\right) \Delta\left|\mathcal{M}^{(i)}\right|_{G \rightarrow q \bar{q}}^{2}
\end{aligned}
$$


where $\mu_{F}$ denotes the factorization mass in the polarized parton densities $\Delta q$ and $\Delta G$. The 3-jet matrix elements $\Delta\left|\mathcal{M}^{(i)}\right|_{q \rightarrow q g}^{2}$ and $\Delta\left|\mathcal{M}^{(i)}\right|_{G \rightarrow q \bar{q}}^{2}$ are given by

$$
\begin{aligned}
& \Delta\left|\mathcal{M}^{(0)}\right|_{q \rightarrow q G}^{2}=C_{F}\left[\frac{x_{p}^{2}+z^{2}}{\left(1-x_{p}\right)(1-z)}+2\left(x_{p}+z\right)\right], \\
& \Delta\left|\mathcal{M}^{(0)}\right|_{G \rightarrow q \bar{q}}^{2}=T_{R}\left(2 x_{p}-1\right)\left(\frac{1}{z}+\frac{1}{1-z}-2\right), \\
& \Delta\left|\mathcal{M}^{(1)}\right|_{q \rightarrow q G}^{2}=4 C_{F} \sqrt{\frac{x_{p} z}{\left(1-x_{p}\right)(1-z)}}\left(1-x_{p}-z\right), \\
& \Delta\left|\mathcal{M}^{(1)}\right|_{G \rightarrow q \bar{q}}^{2}=4 T_{R} \sqrt{\frac{x_{p}\left(1-x_{p}\right)}{z(1-z)}}(1-2 z),
\end{aligned}
$$

where $C_{F}=\frac{4}{3}$ and $T_{R}=\frac{1}{2}$ are the corresponding colour factors. Note that the contribution of the matrix elements $\Delta\left|\mathcal{M}^{(1)}\right|^{2}$ vanish after integration of the angle $\phi$ in (13). Furthermore, the $z$-integration can be done analytically yielding:

$$
\begin{aligned}
\int_{z_{\min }}^{z_{\max }} \int_{0}^{2 \pi} \frac{d \phi}{2 \pi} \Delta\left|M^{(0)}\right|_{q \rightarrow q G}^{2} d z= & C_{F}\left\{-\frac{1+x_{p}^{2}}{1-x_{p}} \ln \frac{z_{\min }}{1-z_{\min }}\right. \\
& \left.+\left(2 x_{p}+1-\frac{3}{2} \frac{1}{1-x_{p}}\right)\left(1-2 z_{\min }\right)\right\} \\
\int_{z_{\min }}^{z_{\max }} \int_{0}^{2 \pi} \frac{d \phi}{2 \pi} \Delta\left|M^{(0)}\right|_{G \rightarrow q \bar{q}}^{2} d z= & -T_{R}\left(2 x_{p}-1\right)\left(\ln \frac{z_{\min }}{1-z_{\min }}+1-2 z_{\min }\right) .
\end{aligned}
$$

\section{FOUR JET CROSS SECTIONS}

In this section we provide exact $\mathcal{O}\left(\alpha_{s}^{2}\right) 4$-jet production cross sections in polarized deep inelastic scattering. The lowest order contributions to the $2 \rightarrow 4$ parton process $l+p \longrightarrow l^{\prime}+p_{1}+p_{2}+p_{3}$ are (the relevant boson-parton subprocesses are depicted in figs. (2a-c)):

$$
\begin{gathered}
l+q \longrightarrow l^{\prime}+q+G+G, \\
l+G \longrightarrow l^{\prime}+q+\bar{q}+G, \\
l+q \longrightarrow l^{\prime}+q+q+\bar{q}
\end{gathered}
$$

and antiquark initiated processes. The corresponding differential partonic cross sections $d \Delta \hat{\sigma}_{p}$ are given by $\left(s_{p}=(p+l)^{2}\right)$

$$
d \Delta \hat{\sigma}_{p}=\frac{1}{2 s_{p}} \frac{\Delta \overline{\left|\mathcal{M}_{i}^{4-j e t}\right|^{2}}}{Q^{4}} d P S^{(4)}
$$


Here $\Delta \overline{\left|\mathcal{M}_{i}^{4-j e t}\right|^{2}}$ denotes the squared matrix element (without the photonpropagator) of the parton processes (23)- (25) including all coupling, statistical and colour factors. The lorentz invariant four particle phase space

$d P S^{(4)}=(2 \pi)^{4} \delta^{4}\left(l+p-l^{\prime}-p_{1}-p_{2}-p_{3}\right) \frac{d^{3} \vec{l}^{\prime}}{(2 \pi)^{3} 2 E^{\prime}} \frac{d^{3} \vec{p}_{1}}{(2 \pi)^{3} 2 E_{1}} \frac{d^{3} \vec{p}_{2}}{(2 \pi)^{3} 2 E_{2}} \frac{d^{3} \vec{p}_{3}}{(2 \pi)^{3} 2 E_{3}}$.

can be parametrized by seven kinematical variables [15], which we choose as $y, Q^{2}, z^{\prime}, \phi, s_{23}, \cos \theta_{23}$ and $\phi_{23}$ where $y$ and $Q^{2}$ are already defined in (2). $s_{23}$ denotes the squared invariant mass of partons 2 and 3

$$
s_{23}=\left(p_{2}+p_{3}\right)^{2}
$$

and the variable $z^{\prime}$ is given by

$$
z^{\prime}=1-\frac{p p_{1}}{p q}
$$

$\phi$ is the azimuthal angle of the outgoing lepton in the (boson-initial parton) center of mass frame. Finally $\theta_{23}$ and $\phi_{23}$ denote the polar and azimuthal angles of parton 2 in the rest frame $\vec{p}_{2}+\vec{p}_{3}=0$ and $\hat{s}=(p+q)^{2}$. In terms of these variables the phase space can be decomposed as [15]

$$
\int_{0}^{1} d \eta \int d P S^{(4)}=\frac{1}{2^{14} \pi^{7}} \int_{0}^{1} d y \int_{0}^{y s} d Q^{2} \int_{x}^{1} d \eta \int_{0}^{1} d z^{\prime} \int_{0}^{2 \pi} d \phi \int_{0}^{z^{\prime} \hat{s}} d s_{23} \int_{-1}^{+1} d \cos \theta_{23} \int_{0}^{2 \pi} d \phi_{23} .
$$

The $\mathcal{O}\left(\alpha_{s}^{2}\right)$ hadronic 4 -jet cross section is then given by

$$
\frac{(2 \pi)^{2} d^{8} \Delta \sigma_{H}}{d y d Q^{2} d \eta d z^{\prime} d \phi d s_{23} d \cos \theta_{23} d \phi_{23}}=\sum_{i=-n_{f}}^{n_{f}}\left(\frac{\alpha_{s}\left(\mu_{R}^{2}\right)}{2 \pi}\right)^{2} \frac{\pi \alpha^{2}}{8 \eta s} \Delta f_{i}\left(\eta, \mu_{F}^{2}\right) \frac{\Delta \overline{\left|\mathcal{M}_{i}^{4-j e t}\right|^{2}}}{Q^{4}}
$$

where the index $i$ refers to the nature of the incoming polarized parton (gluon: $i=0, n_{f}$ light quarks: $i=1, \ldots n_{f}$ and $n_{f}$ light antiquarks: $\left.i=-n_{f}, \ldots-1\right)$. $\mu_{F}$ denotes the factorization mass in the polarized parton densities and $\mu_{R}$ is the renormalization mass entering the strong coupling constant.

In the next section we explicitly specify the squared matrix elements $\Delta \overline{\left|\mathcal{M}_{i}^{4-j e t}\right|^{2}}$ including their respective parton weights $f_{i}\left(\eta, \mu_{F}^{2}\right)$ for the partonic processes (23)(25).

\section{A. $\mathcal{O}\left(\alpha_{s}^{2}\right)$ Four jet matrix elements in polarized $e P$ scattering}

We are now in the position to calculate the squared matrix elements of the hard scattering process. In order to exhibit the parton that initiates the hard 
scattering process we write down the relevant hard scattering process with the parton weights included. We will also explicitly include the colour factors and final state identical particle factors in our expressions. In the following we specify the various quark, antiquark and gluon initiated processes:

(i) Quark and antiquark initiated two quark two gluon processes $\left(q_{f} \rightarrow q_{f} G G\right)$ and $\left(\bar{q}_{f} \rightarrow \bar{q}_{f} G G\right)$

These processes receive contributions from the diagrams of fig. $2 \mathrm{a}$. We divide the contributions into four classes, $|\mathcal{M}|_{A, B, C_{1}, C_{2}}^{2}$ distinguished by their group weight factors (we follow the notation in [17] for $e^{+} e^{-}$jet production):

$$
\begin{array}{ll}
\text { A : } \quad \text { planar abelian graphs: } N_{C} C_{F}^{2} & 11,21,22,31,32,33, \\
& 44,54,55,64,65,66 \\
\text { B : non-planar abelian graphs: } N_{C} C_{F}\left(C_{F}-\frac{N_{C}}{2}\right) & 41,42,43,51,52,53 \\
& 61,62,63 \\
C_{1}: \quad \text { QED-QCD interference graphs: } \frac{1}{2} N_{C}^{2} C_{F} & 71,72,73,74,75,76, \\
& 81,82,83,84,85,86 \\
C_{2}: \quad \text { QCD-QCD graphs: } N_{C}^{2} C_{F} & 77,87,88 .
\end{array}
$$

The squared matrix element is then given by:

$$
\Delta \overline{\left|\mathcal{M}_{q_{f} \rightarrow q_{f} G G}^{4 j e t}\right|^{2}}=\frac{1}{3} \cdot \frac{1}{2}\left(\sum_{f=1}^{n_{f}} e_{f}^{2}\left(\Delta q_{f}+\Delta \bar{q}_{f}\right)\right) \otimes \Delta\left|\mathcal{M}_{q_{f} \rightarrow q_{f} G G}\right|_{A+B+C_{1}+C_{2}}^{2}
$$

The numerical factors in (28) are initial state averaging factor for parton colour and the final state identical particle factor. The symbol $\otimes$ denotes the folding with the parton densities. The numbers $i j$ in the third column refer to the interference of graph $i$ with $j(\equiv(i j), i \geq j)$ in fig. 2a. The explicit expressions for the squared matrix elements $i j$ can be found in the Appendix. They are denoted by $d(i, j)$ and include the color, statistical and average factors.

(ii) Gluon initiated process $\left(G \rightarrow G q_{f} \bar{q}_{f}\right)$

These processes receive contributions from the diagrams of fig. $2 \mathrm{~b}$. The contributions can be divided in similar classes as before (Colour factors and associated graphs are explicitely given):

A : planar abelian graphs:

$$
T_{R} C_{F}\left(N_{C}^{2}-1\right)
$$

$\mathrm{B}$ : non-planar abelian graphs:

$$
T_{R}\left(C_{F}-\frac{N_{C}}{2}\right)\left(N_{C}^{2}-1\right)
$$

$C_{1}$ : QED-QCD interference graphs:

$$
\frac{1}{2} T_{R} N_{C}\left(N_{C}^{2}-1\right)
$$

$C_{2}$ : QCD-QCD graphs: $T_{R} N_{C}\left(N_{C}^{2}-1\right)$
11, 21, 22, 31, 32, 33, $44,54,55,64,65,66$

$41,42,43,51,52,53$

$61,62,63$

$71,72,73,74,75,76$, $81,82,83,84,85,86$

$77,87,88$. 
The squared matrix element reads

$$
\Delta \overline{\left|\mathcal{M}_{G \rightarrow G q_{f} \bar{q}_{f}}^{4 j e t}\right|^{2}}=\frac{1}{8} \cdot\left(\sum_{f^{\prime}=1}^{n_{f^{\prime}}} e_{f^{\prime}}^{2}\right) \Delta G \otimes\left|\mathcal{M}_{G \rightarrow G q_{f} \bar{q}_{f}}\right|_{A+B+C_{1}+C_{2}}^{2}
$$

where $\frac{1}{8}$ is an averaging factor for the initial gluon colours. The numbers ij in the third column refer to the interference of graph $i$ with graph $j$ in fig. $2 \mathrm{~b}$. The explicit expressions for the squared matrix elements $i j$ are to long to be presented in this paper. They are available from the authors.

(iii) Quark and antiquark initiated four-quark process $\left(q_{f} \rightarrow q_{f} q_{f^{\prime}} \bar{q}_{f^{\prime}}\right)$ and $\left(\bar{q}_{f} \rightarrow \bar{q}_{f} q_{f^{\prime}} \bar{q}_{f^{\prime}}\right)$

These diagrams receive contributions from the diagrams in fig. 2c. The contributions are divided in four classes distinguished by their group weight factors:

$$
\begin{array}{lll}
\mathrm{D}: & \text { non-interference contributions: } N_{C} C_{F} T_{R} & 11,21,22,55,65,66 \\
\mathrm{D}^{\prime}: \text { non-interference contributions: } N_{C} C_{F} T_{R} & 33,43,44,77,87,88 \\
\mathrm{E}: \text { non-interference contributions: } & 31,32,41,42,51,52, \\
& { }_{N} C_{F}\left(C_{F}-\frac{N_{C}}{2}\right) & 61,62,73,74,75,76, \\
& 83,84,85,86 \\
\text { F : interference contributions: } N_{C} C_{F} T_{R} & 53,54,63,64,71,72, \\
& 81,82 .
\end{array}
$$

The squared matrix element reads

$$
\begin{aligned}
\Delta \overline{\left|\mathcal{M}^{4 j e t}\right|_{q \rightarrow q q \bar{q}}^{2}}= & \frac{1}{3} \cdot \frac{1}{2}\left[\sum_{f=1}^{n_{f}} e_{f}^{2}\left(\Delta q_{f}+\Delta \bar{q}_{f}\right) \otimes\left(n_{f^{\prime}} \cdot|\mathcal{M}|_{D}^{2}+|\mathcal{M}|_{E}^{2}\right)\right. \\
& +\sum_{f=1}^{n_{f}}\left(\sum_{f^{\prime}=1}^{n_{f^{\prime}}} e_{f^{\prime}}^{2}\right)\left(\Delta q_{f}+\Delta \bar{q}_{f}\right) \otimes|\mathcal{M}|_{D^{\prime}}^{2} \\
& \left.+\sum_{f=1}^{n_{f}}\left(\sum_{f^{\prime}=1}^{n_{f^{\prime}}} e_{f^{\prime}}\right) e_{f}\left(\Delta q_{f}-\Delta \bar{q}_{f}\right) \otimes|\mathcal{M}|_{F}^{2}\right]
\end{aligned}
$$

( $\frac{1}{3}$ colour averaging, $\frac{1}{2}$ statistical factor for identical particles in the final state). The numbers $i j$ in the third column refer to the interference of graph $i$ with graph $j$ in fig. 2c. The explicit expressions for the squared matrix elements $i j$ can be found in the Appendix. They are denoted by $e(i, j)$ and include the color, statistical and average factors.

Some comments are in order for this subprocess: We do not distinguish between equal and unequal flavour cases. The overcounting in the unequal flavour case $\left(f \neq f^{\prime}\right)$ is compensated by our multiplication of these contribution with the Fermi statistical factor $\frac{1}{2}$ as in the equal flavour case $\left(f=f^{\prime}\right)$.

The interference contributions class $F$ involve the calculation of two fermion 
traces. They involve a product of traces of 3 fermion (or antifermion) propagators. Each such trace is anti-symmetric under quark-antiquark exchange for the vector current $\gamma_{\mu}$ due to charge conjugation invariance. Note that the interference class $F$ does not contribute to the total cross section if one does not identify the flavours of the produced quarks and antiquarks. This is a consequence of charge conjugation - the non-abelian generalization of Furry's theorem.

\section{POLARIZED PARTON DENSITIES}

The hadronic 3- and 4-jet cross sections are obtained by folding the partonic cross sections with the polarized parton densities (see (13,27)). In this section, we review the set of the polarized parton density parametrization 13 which we will use in our numerical calculation. The sea and gluon parton densities are chosen in such a way that the experimental result for the Ellis-Jaffe sum rule is reproduced up to $\mathcal{O}\left(\alpha_{s}\right)$.

These densities are given by:

$$
\begin{aligned}
& \Delta u_{V}(x)=\alpha(x) u_{V}\left(x, M^{2}\right) \\
& \Delta d_{V}(x)=\beta(x) d_{V}\left(x, M^{2}\right)
\end{aligned}
$$

with

$$
\alpha(x)=x^{0.287}, \quad \beta(x)=\left(\frac{x-x_{0}}{1-x_{0}}\right) z_{p}
$$

with $x_{0}=0.75$ and $p=0.76$. The $u_{V}\left(x, M^{2}\right)$ and $d_{V}\left(x, M^{2}\right)$ are the unpolarized parton densities given by the DFLM4 set $(\Lambda=0.2 \mathrm{GeV})$ at $M^{2}=10 \mathrm{GeV}^{2}$ [16]. According to [13] we can adopt the following parametrization:

set 1: At $M^{2}=10 \mathrm{GeV}^{2}$ one has a large negative polarized sea-quark density and a zero polarized gluon density

$$
\begin{aligned}
\Delta s(x) & =-11.8 x^{0.94}(1-x)^{5} s\left(x, M^{2}\right), \\
\Delta G(x) & =0
\end{aligned}
$$

set 2: At $M^{2}=10 \mathrm{GeV}^{2}$ one has a zero polarized sea-quark density and a large positive gluon density

$$
\begin{aligned}
\Delta s(x) & =0 \\
\Delta G(x) & =6.0 x^{0.76}(1-x)^{3} G\left(x, M^{2}\right) .
\end{aligned}
$$

The above gluon density is about the same as the one proposed in [5]. 
set 3: In a realistic situation it is unlikely that $\Delta s(x)$ or $\Delta G(x)$ vanishes at some scale for all $x$. Therefore the authors in [13 also proposed

$$
\begin{aligned}
\Delta s(x) & =-3.39 x^{0.62}(1-x)^{1.4} s\left(x, M^{2}\right), \\
\Delta G(x) & =2.69 x^{0.76}(1-x)^{3} G\left(x, M^{2}\right),
\end{aligned}
$$

for $M^{2}=10 \mathrm{GeV}^{2}$.

$s\left(x, M^{2}\right)$ and $G\left(x, M^{2}\right)$ represent the unpolarized parton densities belonging to the DFLM4 set [16].

\section{NUMERICAL RESULTS}

We will now turn to our numerical cross section results. In order to define the deep inelastic scattering region, we choose the lower bounds

$$
\begin{aligned}
Q^{2} & \geq Q_{0}^{2}=4 \mathrm{GeV}^{2}, \\
W^{2} & \geq W_{0}^{2}=5 \mathrm{GeV}^{2}, \\
x & \geq x_{0} \equiv 10^{-2} .
\end{aligned}
$$

We introduce a limit in $W^{2}$ to insure an appropriate hadronic final state. When producing well separated multijet final states the effective lower limit of $W^{2}$ will actually be increased (see $(39,40)$ ). As already discussed in section III one encounters infrared/mass (IR/M) singularities when calculating the $\mathcal{O}\left(\alpha_{s}\right)$ and $\mathcal{O}\left(\alpha_{s}^{2}\right)$ tree graph cross sections, which originates from the emission of soft and/or collinear partons. The tree graph cross sections make sense only for the production of well separated hard jets. We introduce the following invariant mass-cut-off to

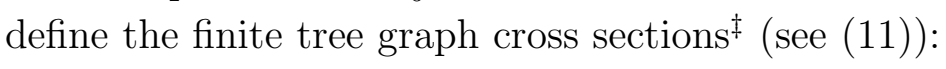

$$
s_{i j} \geq \max \left\{y_{c} W^{2}, M_{0}^{2}\right\}, \quad s_{i j} \equiv\left(p_{i}+p_{j}\right)^{2} \quad i \neq j=1,2,3,(4)
$$

where $y_{c}$ is the resolution parameter for which we choose $y_{c}=0.04$ [14, 15. $M_{0}$ is an additional fixed mass cut which we have introduced in order to clearly separate the perturbative and non-perturbative regime in the case, where $W^{2}$ is small. Note that $W^{2}$ is limited from below by $W_{0}^{2}$ (see (36)). This leads to too small invariant masses for a perturbative calculation to be meaningful. For example with $y_{c}=0.04$ one obtains $s_{i j} \leq 0.2 \mathrm{GeV}^{2}$. A reasonable choice for the

\footnotetext{
${ }^{\ddagger}$ Note, that the remnant jet $\left(p_{3}\right.$ in $\mathcal{O}\left(\alpha_{s}\right)$ and $p_{4}$ in $\left.\mathcal{O}\left(\alpha_{s}^{2}\right)\right)$ is included in this definition.
} 
fixed mass cut in (37) is $M_{0}=2 \mathrm{GeV}$ [15]. Our subsequent analysis is then based on the invariant mass cut

$$
s_{i j} \geq \max \left\{0.04 W^{2}, 4 \mathrm{GeV}^{2}\right\} .
$$

The invariant mass cut in (38) introduces then the following available ranges in $\eta$ and $W^{2}$ for the $\mathcal{O}\left(\alpha_{s}\right)$ 3-jet and $\mathcal{O}\left(\alpha_{s}^{2}\right)$ 4-jet cross sections:

$\underline{\mathcal{O}\left(\alpha_{s}\right) 3 \text {-jet case: }}$

$$
\begin{aligned}
& W^{2} \geq \max \left\{W_{0}^{2}, 3 M_{0}^{2}\right\}=12 \mathrm{GeV}^{2} \\
& \eta \geq y_{c}+x\left(1-y_{c}\right)
\end{aligned}
$$

$\underline{\mathcal{O}\left(\alpha_{s}^{2}\right) \text { 4-jet case: }}$

$$
\begin{gathered}
W^{2} \geq \max \left\{W_{0}^{2}, 6 M_{0}^{2}\right\}=24 \mathrm{GeV}^{2} \\
\eta \geq 3 y_{c}+x\left(1-3 y_{c}\right)
\end{gathered}
$$

As can be seen from (39) and (40), the lower bound in $\eta$ and $W^{2}$ increases with jet multiplicity.

In our numerical calculation we use the one-loop formula of the strong coupling constant

$$
\alpha_{s}\left(\mu_{R}^{2}\right)=\frac{12 \pi}{\left(33-2 n_{f}\right) \ln \frac{\mu_{R}^{2}}{\Lambda^{2}}}
$$

and the $\Lambda$ value consistent to our choise of polarized parton distributions. The renormalization scale $\mu_{R}^{2}$ and the factorization scale $\mu_{F}^{2}$ are both fixed to $Q^{2}$. The value of $\alpha_{s}$ is matched at the thresholds $q=m_{q}$ and the number of flavours $n_{f}$ in $\alpha_{s}$ is fixed by the number of flavours for which the masses are less than $Q^{2}$. Furthermore the number of quark flavours that can be pair-produced are set equal to $n_{f}$ chosen in $\alpha_{s}$.

In figs. 3 a-f we show how the various cross sections vary with a given invariant energy $\sqrt{s}$ for the three different sets of parametrizations (set 1-3) of the polarized parton densities. The $Q^{2}$ range is chosen as $5 \mathrm{GeV}^{2} \leq Q^{2} \leq s$. One observes, that all quark initiated subprocesses are positiv and have a maximum around $\sqrt{s}=15-20 \mathrm{GeV}$. In contrast, the gluon initiated contribution to $\Delta \sigma$ is negativ and decreasing with increasing $\sqrt{s}$.

In figs. 4-7 we show the dependence of the $\mathcal{O}\left(\alpha_{s}\right)$ 3-jet cross sections on the basic kinematical variables $x$ and $W^{2}$ for the different parametrizations. We have restricted the $Q^{2}$ range from $5 \mathrm{GeV}^{2}<Q^{2}<7 \mathrm{GeV}^{2}$ (figs. 4,6) and $9 \mathrm{GeV}^{2}<$ $Q^{2}<11 \mathrm{GeV}^{2}$ (figs. 5,7). The quark (dashed) and gluon (dotted) initiated cross sections are shown separately as well as the sum (solid). 
Comparing figs. 4a-c (5a-c) one observes large differences in the $x$ distribution using different parametrizations. In particular, figs. 4b (5b) show that the 3 -jet cross section (solid curve) is negative for a large gluon contribution for $x$ values less than about 0.04 . An analysis of the $x$-dependence at lower $x$ values is therefore very sensitive to the polarized gluon densities.

Figs. 6a-c (7a-c) show the $W$-distribution of 3-jet production for the three different sets of parametrizations, where the $Q^{2}$ range is given by $5 \mathrm{GeV}^{2}<Q^{2}<$ $7 \mathrm{GeV}^{2}\left(9 \mathrm{GeV}^{2}<Q^{2}<11 \mathrm{GeV}^{2}\right)$. Here larger values of $W$ are more sensitive to the choice of polarized parton distributions. The gluon process gives a negative contribution and dominates the resulting cross section in the large $W$ range in figs $6 \mathrm{~b}, \mathrm{c}$. This is a reflection of the $x$ behaviour since $W^{2}=(1-x) y s$. Like for the $x$-distribution, the predictions for the two extreme choices of parton distributions are very different.

Figs. 8 and 9 show predictions of the $x$ and $W$ dependence of the $O\left(\alpha_{s}^{2}\right)$ 4jet cross sections for the three different sets of parametrizations and $5 \mathrm{GeV}^{2}<$ $Q^{2}<7 \mathrm{GeV}^{2}$. The behaviour of the $x$ and $W$ dependence is similar to the $O\left(\alpha_{s}\right)$ 3 -jet cross section. One observes that the $q \rightarrow q G G$ subprocess dominates the 4 quark process $q \rightarrow q q \bar{q}$ over the whole $x$ and $W$ ranges. However, for set 2 of the parton distributions (fig. 8b), the gluon process $G \rightarrow q \bar{q} G$ gives a large negative contribution for smaller $x$ values, which gives again a negativ cross section for the sum of all contributions. The sum of the contributions for set 3 of the parton distributions (figs. 8c, 9c) remains still positive indicating that the 4-jet cross sections are less sensitive to the different parametrizations. The behaviour of the $W$ dependence in the 4-jet distributions is again similar to the behaviour found for the 3 -jet cross setcions. The gluon process gives a negative contribution at larger $W$ values.

\section{CONCLUSIONS}

Jet production in DIS scattering experiments, using polarized beams and polarized targets, has been studied. It is shown that the jet cross sections are strongly dependent on the particular form of the spin dependent parton distributions used in the calculation. Therefore, jet production in polarized DIS can be used to put rigid constraints on the various parametrizations of polarized structure functions. These informations allow to discriminate between the different physical pictures of the proton spin underlying these parametrizations.

\section{Acknowledgements}

This work is supported in part by the U.S. Department of Energy under contract No. DE-AC02-76ER00881, in part by BMFT Contract 055KA94P1, and in part 
by the University of Wisconsin Research Committee with funds granted by the Wisconsin Alumni Research Foundation. 


\section{APPENDIX A: MATRIX ELEMENTS}

We give here explicit formulae for the squared matrix elements $\Delta \overline{\left|\mathcal{M}_{i}^{4-j e t}\right|^{2}}$ in (27- 30). As usual, $\Delta \overline{\left|\mathcal{M}_{i}^{4-j e t}\right|^{2}}$ can be written in terms of the lepton tensor $L_{\mu \nu}$ and the hadron tensor $\Delta H^{\mu \nu}=\frac{1}{2}\left(H^{\mu \nu+}-H^{\mu \nu-}\right)$, where $H^{\mu \nu}+\left(H^{\mu \nu-}\right)$ denotes the hadron tensor for the process when the incoming parton has positiv (negativ) helicity. The hadron tensor $\Delta H^{\mu \nu}$ can be expanded in terms of the following tensors:

$$
\begin{aligned}
{\left[p, p_{1}\right] } & :=\varepsilon^{\mu \nu \alpha \beta} p_{\alpha} p_{1 \beta} \\
{\left[p, p_{2}\right] } & :=\varepsilon^{\mu \nu \alpha \beta} p_{\alpha} p_{2 \beta} \\
{\left[p, p_{3}\right] } & :=\varepsilon^{\mu \nu \alpha \beta} p_{\alpha} p_{3 \beta} \\
{\left[p_{1}, p_{2}\right] } & :=\varepsilon^{\mu \nu \alpha \beta} p_{1 \alpha} p_{2 \beta} \\
{\left[p_{1}, p_{3}\right] } & :=\varepsilon^{\mu \nu \alpha \beta} p_{1 \alpha} p_{3 \beta} \\
{\left[p_{2}, p_{3}\right] } & :=\varepsilon^{\mu \nu \alpha \beta} p_{2 \alpha} p_{3 \beta} .
\end{aligned}
$$

Furthermore, we introduce the following notation

$$
\begin{aligned}
s & :=p p_{3} \\
t & :=p_{2} p_{3} \\
u & :=p p_{2} \\
x & :=p_{1} p_{3} \\
y & :=p p_{1} \\
z & :=p_{1} p_{2} .
\end{aligned}
$$

To obtain the full matrix elements $\Delta \overline{\left|\mathcal{M}_{i}^{4-j e t}\right|^{2}}$, the corresponding hadron tensors for each subprocess have to be contracted with the anti-symmetric part of the the lepton tensor $L_{\mu \nu}^{A}$ :

$$
L_{\mu \nu}^{A}=2 i \epsilon_{\mu \nu \alpha \beta} l^{\alpha} l^{\beta}
$$

\section{Matrix elements for $\left(q_{f} \rightarrow q_{f} G G\right)$ and $\left(\bar{q}_{f} \rightarrow \bar{q}_{f} G G\right)$}

The results for the interference of graph $i$ with graph $j$ in fig. $2 \mathrm{a}$ is denoted by $d(i, j)$. Note that $d(i, j)$ contain the color, statistical and average factors as given in (28).

$$
\begin{aligned}
d(1,1)= & -16 i\left[p_{2}, p_{3}\right] /(9 s z) \\
d(2,1)= & 16 i\left(\left[p, p_{1}\right] u+\left[p, p_{2}\right](s-x+y+2 u-t)+\left[p, p_{3}\right] u\right. \\
& \left.+\left[p_{1}, p_{3}\right] u+\left[p_{2}, p_{3}\right] u\right) /(9 s z(x+z+t)) \\
d(2,2)= & -16 i\left(-\left[p, p_{1}\right](z+t)+\left[p, p_{2}\right] x-\left[p, p_{3}\right](z+t)\right) /\left(9 z(x+z+t)^{2}\right)
\end{aligned}
$$




$$
\begin{aligned}
& d(3,1)=16 i\left(\left[p, p_{1}\right] x+\left[p, p_{2}\right] x+\left[p_{1}, p_{2}\right] x+\left[p_{1}, p_{3}\right](2 x-y+z-u+t)\right. \\
& \left.+\left[p_{2}, p_{3}\right] x\right) /(9 s z(s+u-t)) \\
& d(3,2)=16 i\left([ p , p _ { 1 } ] \left(s y+s u+x^{2}-3 x y+x z-2 x u+2 x t+2 y^{2}-y z+3 y u\right.\right. \\
& \left.-2 y t+u^{2}-2 u t+t^{2}\right)+\left[p, p_{2}\right]\left(-2 x y-x u+y^{2}-y z+y u\right) \\
& +\left[p, p_{3}\right]\left(-x y+y^{2}+y u-y t+z u-z t\right)+\left[p_{1}, p_{2}\right](s x+s t-x y \\
& \left.+y^{2}+y u-y t\right)+\left[p_{1}, p_{3}\right]\left(s y-x y-x u+y^{2}+2 y u\right) \\
& \left.+\left[p_{2}, p_{3}\right](s y+s z-x y-x u-y z+y u)\right) /(9 s z(s+u-t)(x+z+t)) \\
& d(3,3)=16 i\left(\left[p, p_{1}\right](s-t)+\left[p_{1}, p_{2}\right](s-t)-\left[p_{1}, p_{3}\right] u\right) /\left(9 s(s+u-t)^{2}\right) \\
& d(4,1)=-2 i\left(\left[p, p_{1}\right] y(s-x+2 y-z+u)+\left[p, p_{2}\right] y(s-x+y)\right. \\
& +\left[p, p_{3}\right] y(y-z+u)+\left[p_{1}, p_{2}\right] y(s-x+y) \\
& \left.+\left[p_{1}, p_{3}\right] y(y-z+u)\right) /(9 s x z u) \\
& d(4,2)=2 i\left(\left[p, p_{1}\right](s z+z u+u t)+\left[p, p_{2}\right](s z+x z-x u+y z)\right. \\
& +\left[p, p_{3}\right](s z-2 x y+x z-x u-y z-y t)+\left[p_{1}, p_{2}\right](-x u+y t) \\
& \left.-\left[p_{1}, p_{3}\right] x u+\left[p_{2}, p_{3}\right] y(x+z)\right) /(9 x z u(x+z+t)) \\
& d(4,3)=2 i\left(\left[p, p_{1}\right](s x+s z-x t)-\left[p, p_{2}\right] x u+\left[p, p_{3}\right](-x u+y t)\right. \\
& +\left[p_{1}, p_{2}\right](s y+s z+s u-x u+2 y u-y t)+\left[p_{1}, p_{3}\right](-s y+s z \\
& \left.+s u-x u)-\left[p_{2}, p_{3}\right] y(s+u)\right) /(9 s x u(s+u-t)) \\
& d(5,2)=4 i\left(-\left[p, p_{1}\right] t+\left[p, p_{2}\right](x+z)+\left[p, p_{3}\right](x+z)\right) /(9 x z(x+z+t)) \\
& d(5,3)=-2 i\left([ p , p _ { 1 } ] \left(s^{2}-2 s x+3 s y-s z+s u-2 s t+x^{2}-3 x y+x z\right.\right. \\
& \left.-x u+2 x t+2 y^{2}-y z+y u-2 y t\right)+\left[p, p_{2}\right] y(s-x+y) \\
& +\left[p, p_{3}\right] y(s-x+y)+\left[p_{1}, p_{2}\right] y(s-x+y) \\
& \left.+\left[p_{1}, p_{3}\right] y(s-x+y)\right) /(9 s x(x+z+t)(s+u-t)) \\
& d(6,3)=4 i\left(\left[p, p_{1}\right] t+\left[p_{1}, p_{2}\right](s+u)+\left[p_{1}, p_{3}\right](s+u)\right) /(9 s u(s+u-t)) \\
& d(7,1)=i\left([ p , p _ { 1 } ] \left(2 s x+s z+s t-x^{2}+2 x y-2 x z+x u-x t+4 y z+2 y t\right.\right. \\
& +2 z u-z t-u t)+\left[p, p_{2}\right]\left(s x+2 s z-x^{2}+x y-x z+2 x u-x t\right. \\
& +2 y z+y t+4 z u-2 z t)+\left[p, p_{3}\right](-s z+2 x y+2 x z \\
& +2 x u+y z+2 z u)+2\left[p_{1}, p_{2}\right] s(x+t) \\
& +\left[p_{1}, p_{3}\right](-2 s z+x y-x u+2 y z+3 y t+z u-u t) \\
& \left.+\left[p_{2}, p_{3}\right](y z+2 y t+2 z u)\right) /(s z t(x+z+t)) \\
& d(7,2)=2 i\left(\left[p, p_{1}\right]\left(-x z-x t+z^{2}-2 z t-t^{2}\right)+\left[p, p_{2}\right]\left(x^{2}+x t+z^{2}-z t\right)\right. \\
& \left.+2\left[p, p_{3}\right]\left(x^{2}+2 x z+x t+2 z^{2}+z t\right)\right) /\left(z t(x+z+t)^{2}\right)
\end{aligned}
$$




$$
\begin{aligned}
& d(7,3)=-i\left([ p , p _ { 1 } ] \left(s^{2}-s x+2 s y+2 s z+3 x u-2 y u-2 y t+2 z u-z t\right.\right. \\
& \left.-u^{2}+4 u t-2 t^{2}\right)+\left[p, p_{2}\right](s y+2 x u-y u-3 y t) \\
& +\left[p, p_{3}\right](s y-y u+y t-2 z u+2 z t)+\left[p_{1}, p_{2}\right](-3 s x-s z-3 s t \\
& -2 y u+2 y t)+\left[p_{1}, p_{3}\right](-s y+3 x u-3 y u-y t+z u+u t) \\
& \left.+\left[p_{2}, p_{3}\right](-s y-2 s z+2 x u-y u-3 y t)\right) /(s t(s+u-t)(x+z+t)) \\
& d(7,7)=-i\left(\left[p, p_{1}\right]\left(2 x^{2}+14 x z+9 x t+4 z^{2}+11 z t-t^{2}\right)+\left[p, p_{2}\right]\left(-5 x^{2}+3 x z\right.\right. \\
& \left.+3 x t+8 z t)+\left[p, p_{3}\right]\left(5 x z+8 x t-3 z^{2}+5 z t\right)\right) /\left(t^{2}(x+z+t)^{2}\right) \\
& d(8,1)=-i\left(\left[p, p_{1}\right](2 s x-4 s y+s z-2 s u+s t+x u+x t-2 y u+2 y t+2 z u\right. \\
& \left.-z t-u^{2}+u t\right)+\left[p, p_{2}\right](s x-2 s y-2 s z-x u+x t-y u+3 y t) \\
& +2\left[p, p_{3}\right] z(u-t)+\left[p_{1}, p_{2}\right](2 s x-s y-s z+2 s u+2 x u-2 y u) \\
& +\left[p_{1}, p_{3}\right]\left(4 s x-2 s y+2 s z-s u+2 s t+2 x u-y u+y t+z u-u^{2}+u t\right) \\
& \left.+\left[p_{2}, p_{3}\right](2 s x-s y+2 y t)\right) /(s z t(s+u-t)) \\
& d(8,2)=i\left([ p , p _ { 1 } ] \left(2 s x+2 s z+s t-x^{2}+2 x y+3 x u-4 x t-2 y z-2 y t+z^{2}\right.\right. \\
& \left.-z u-2 t^{2}\right)+\left[p, p_{2}\right](s x+3 x y+3 x u-x t+y z-y t)+\left[p, p_{3}\right](-s z \\
& +2 x y+2 y t-3 z u+3 z t)+\left[p_{1}, p_{2}\right](-2 s x-2 s t+x y-y z+y t) \\
& \left.+\left[p_{1}, p_{3}\right](x y+2 x u-y z-3 y t)+\left[p_{2}, p_{3}\right](-2 s z+x y+2 x u+y z-3 y t)\right) \\
& \cdot 1 /(z t(s+u-t)(x+z+t)) \\
& d(8,3)=-2 i\left(\left[p, p_{1}\right]\left(s^{2}-s u+2 s t+u t-t^{2}\right)+2\left[p_{1}, p_{2}\right]\left(2 s^{2}+2 s u-s t+u^{2}-u t\right)\right. \\
& \left.+\left[p_{1}, p_{3}\right]\left(s^{2}+s t+u^{2}-u t\right)\right) /\left(s t(s+u-t)^{2}\right) \\
& d(8,7)=-i\left(\left[p, p_{1}\right]\left(4 s x+14 s z+s t+14 x u-x t-16 y t+z t-u t+2 t^{2}\right)\right. \\
& +\left[p, p_{2}\right](-5 s x+7 x u+5 x t-8 y t)+\left[p, p_{3}\right](5 s z-8 y t-7 z u+7 z t) \\
& +\left[p_{1}, p_{2}\right](-5 s x+7 s z-5 s t-8 y t)+\left[p_{1}, p_{3}\right](5 x u-8 y t-7 z u-7 u t) \\
& \left.+12\left[p_{2}, p_{3}\right](-s z+x u)\right) /\left(t^{2}(s+u-t)(x+z+t)\right) \\
& d(8,8)=-i\left(\left[p, p_{1}\right]\left(2 s^{2}+14 s u-9 s t+4 u^{2}-11 u t-t^{2}\right)\right. \\
& \left.+\left[p_{1}, p_{2}\right]\left(-5 s^{2}+3 s u-3 s t-8 u t\right)+\left[p_{1}, p_{3}\right]\left(5 s u-8 s t-3 u^{2}-5 u t\right)\right) \\
& \cdot 1 /\left(t^{2}(s+u-t)^{2}\right) \text {. }
\end{aligned}
$$

The remaining matrix elements can be obtained by the interchange of $p_{2} \leftrightarrow p_{3}, s \leftrightarrow u, x \leftrightarrow z$ from the above results.

$$
\begin{aligned}
& d(4,4) \longleftrightarrow d(1,1) \quad d(5,4) \longleftrightarrow d(2,1) \quad d(5,5) \longleftrightarrow d(2,2) \\
& d(6,4) \longleftrightarrow d(3,1) \quad d(6,5) \longleftrightarrow d(3,2) \quad d(6,6) \longleftrightarrow d(3,3) \\
& d(5,1) \longleftrightarrow d(4,2) \quad d(6,1) \longleftrightarrow d(4,3) \quad d(6,2) \longleftrightarrow d(5,3) \\
& d(7,4) \longleftrightarrow d(7,1) \quad d(7,5) \longleftrightarrow d(7,2) \quad d(7,6) \longleftrightarrow d(7,3) \\
& d(8,4) \longleftrightarrow d(8,1) \quad d(8,5) \longleftrightarrow d(8,2) \quad d(8,6) \longleftrightarrow d(8,3) .
\end{aligned}
$$




\section{Matrix elements for $\left(G \rightarrow G q_{f} \bar{q}_{f}\right)$}

The result for the interference of graph $i$ with graph $j$ in fig. $2 \mathrm{~b}$ is denoted by $c(i, j)$. The analytical results are to long to present here. They are available from the authors.

\section{Matrix elements for $\left(q_{f} \rightarrow q_{f} q_{f^{\prime}} \bar{q}_{f^{\prime}}\right)$ and $\left(\bar{q}_{f} \rightarrow \bar{q}_{f} q_{f^{\prime}} \bar{q}_{f^{\prime}}\right)$}

The results for the interference of graph $i$ with graph $j$ in fig. 2c is denoted by $e(i, j)$. Note that $e(i, j)$ contain the color, statistical and average factors as given in (30).

$$
\begin{aligned}
& e(1,1)=-2 i\left(\left[p, p_{1}\right](-2 x z-x t-z t)+\left[p, p_{2}\right]\left(x^{2}-x z-z t\right)\right. \\
& \left.+\left[p, p_{3}\right]\left(-x z-x t+z^{2}\right)\right) /\left(3 t^{2}(x+z+t)^{2}\right) \\
& e(2,1)=2 i\left(2\left[p, p_{1}\right](-s z-x u+y t)+\left[p, p_{2}\right](s x-x t-x u+y t)\right. \\
& +\left[p, p_{3}\right](-s z+y t-z t+z u)+\left[p_{1}, p_{2}\right](s x-s z+s t+y t) \\
& +\left[p_{1}, p_{3}\right](-x u+y t+z u+t u) \\
& \left.+2\left[p_{2}, p_{3}\right](s z-x u)\right) /\left(3 t^{2}(x+z+t)(s-t+u)\right) \\
& e(2,2)=-2 i\left(\left[p, p_{1}\right](s t-2 s u+t u)+\left[p_{1}, p_{2}\right]\left(s^{2}-s u+t u\right)\right. \\
& \left.+\left[p_{1}, p_{3}\right]\left(s t-s u+u^{2}\right)\right) /\left(3 t^{2}(s-t+u)^{2}\right) \\
& e(3,1)=2 i\left(\left[p, p_{1}\right] z(x+t)+\left[p, p_{2}\right] z(x+t)-\left[p_{1}, p_{3}\right] z(y+u)\right. \\
& \left.-\left[p_{2}, p_{3}\right] z(y+u)\right) /(9 t u(x+z+t)(y-z+u)) \\
& e(3,2)=2 i\left(\left[p, p_{1}\right](-x u+y t)+\left[p, p_{2}\right](-s z-x u+y t)+\left[p, p_{3}\right] z(-t+u)\right. \\
& +\left[p_{1}, p_{2}\right] s(y+u)+\left[p_{1}, p_{3}\right](s z-x u+y t) \\
& \left.+\left[p_{2}, p_{3}\right](-x u+y t)\right) /(9 t u(s-t+u)(y-z+u)) \\
& e(3,3)=2 i\left(\left[p, p_{3}\right]\left(-y z+y u+z^{2}\right)-\left[p_{1}, p_{3}\right] u(y+z)\right. \\
& \left.+\left[p_{2}, p_{3}\right]\left(y^{2}-y z-z u\right)\right) /\left(3 u^{2}(y-z+u)^{2}\right) \\
& e(4,1)=2 i\left(\left[p, p_{1}\right](-s z-x u+y t)+\left[p, p_{2}\right](-x u+y t)+\left[p, p_{3}\right] z(-t+u)\right. \\
& +\left[p_{1}, p_{2}\right] s(x+t)+\left[p_{1}, p_{3}\right](-x u+y t) \\
& \left.+\left[p_{2}, p_{3}\right](s z-x u+y t)\right) /(9 t u(x+z+t)(s-t+u)) \\
& e(4,2)=-4 i\left(\left[p, p_{1}\right] s(t-u)+\left[p_{1}, p_{2}\right] s^{2}+\left[p_{1}, p_{3}\right] s(t-u)\right) /\left(9 t u(s-t+u)^{2}\right) \\
& e(4,3)=2 i\left(\left[p, p_{1}\right](-x u+y t-z t+t u)-2\left[p, p_{2}\right] x u\right. \\
& +\left[p, p_{3}\right](s z-x u-z t+z u)+\left[p_{1}, p_{2}\right](s y-s z+s u+x u) \\
& \left.+\left[p_{2}, p_{3}\right](-s y-x u+y t-y u)\right) /\left(3 u^{2}(s-t+u)(y-z+u)\right)
\end{aligned}
$$




$$
\begin{aligned}
& e(4,4)=-2 i\left(\left[p, p_{1}\right]\left(s t-s u-t^{2}\right)+\left[p_{1}, p_{2}\right]\left(s^{2}-s t-t u\right)\right. \\
& \left.-\left[p_{1}, p_{3}\right] u(s+t)\right) /\left(3 u^{2}(s-t+u)^{2}\right) \\
& e(5,1)=-4 i\left(\left[p, p_{1}\right] z(x+t)+\left[p, p_{2}\right] z(x+t)-\left[p, p_{3}\right] z^{2}\right) /\left(9 x t(x+z+t)^{2}\right) \\
& e(5,2)=-2 i\left(\left[p, p_{1}\right](-s z-x u+y t)+\left[p, p_{2}\right](-x u+y t)+\left[p, p_{3}\right] z(-t+u)\right. \\
& +\left[p_{1}, p_{2}\right] s(x+t)+\left[p_{1}, p_{3}\right](-x u+y t) \\
& \left.+\left[p_{2}, p_{3}\right](s z-x u+y t)\right) /(9 x t(x+z+t)(s-t+u)) \\
& e(5,3)=2 i\left(\left[p, p_{1}\right](-x z-2 z t+t u)+\left[p, p_{2}\right](s z-x z-x u-z t)\right. \\
& +\left[p, p_{3}\right](s z-x u-y t)+\left[p_{1}, p_{2}\right](-s z+x u-y t) \\
& +\left[p_{1}, p_{3}\right](-s z+x u+y z+z u) \\
& \left.+\left[p_{2}, p_{3}\right](-x y+z u)\right) /(3 x u(x+z+t)(y-z+u)) \\
& e(5,4)=-2 i\left(\left[p, p_{1}\right](-s z-x u+y t)+\left[p, p_{2}\right](-x t-x u+y t-z t)\right. \\
& +\left[p, p_{3}\right](-x t-2 z t+z u)+\left[p_{1}, p_{2}\right](s x-t u) \\
& +\left[p_{1}, p_{3}\right](-s t-x u \\
& \left.+y t-t u)+\left[p_{2}, p_{3}\right](s z-x u+y t)\right) /(3 x u(x+z+t)(s-t+u)) \\
& e(6,2)=-2 i\left(\left[p, p_{1}\right] s(t-u)+\left[p, p_{2}\right] s(x-y)+\left[p_{1}, p_{3}\right] s(t-u)\right. \\
& \left.+\left[p_{2}, p_{3}\right] s(x-y)\right) /(9 x t(s-t+u)(s-x+y)) \\
& e(6,3)=-2 i\left(\left[p, p_{1}\right](-s z+x u-y t)+\left[p, p_{2}\right](s y-x y+x u-y t)\right. \\
& +\left[p, p_{3}\right](-x z+y u)+\left[p_{1}, p_{2}\right](-2 s y-s u+x y) \\
& +\left[p_{1}, p_{3}\right](x u+y z-y t-y u) \\
& \left.+\left[p_{2}, p_{3}\right](s z+x u-y t)\right) /(3 x u(y-z+u)(s-x+y)) \\
& e(6,4)=-2 i\left(\left[p, p_{1}\right](-s u+x t)+\left[p, p_{2}\right](s x-s y+s z-x u)\right. \\
& +\left[p, p_{3}\right](s z-x u+y t)+\left[p_{1}, p_{2}\right](-s z+x u+y t) \\
& +\left[p_{1}, p_{3}\right](-s z+s t-s u+x u) \\
& \left.+\left[p_{2}, p_{3}\right](s x-2 s y-y u)\right) /(3 x u(s-t+u)(s-x+y)) \\
& e(7,3)=4 i\left(-\left[p, p_{3}\right] z^{2}+\left[p_{1}, p_{3}\right] z(y+u)+\left[p_{2}, p_{3}\right] z(y+u)\right) /\left(9 y u(y-z+u)^{2}\right) \\
& e(7,4)=-2 i\left(\left[p, p_{1}\right](-x u+y t)+\left[p, p_{2}\right](-s z-x u+y t)\right. \\
& +\left[p, p_{3}\right] z(-t+u)+\left[p_{1}, p_{2}\right] s(y+u)+\left[p_{1}, p_{3}\right](s z-x u+y t) \\
& \left.+\left[p_{2}, p_{3}\right](-x u+y t)\right) /(9 y u(s-t+u)(y-z+u)) \\
& e(8,4)=-2 i\left(\left[p, p_{1}\right] s(t-u)+\left[p, p_{2}\right] s(x-y)+\left[p_{1}, p_{3}\right] s(t-u)\right. \\
& \left.+\left[p_{2}, p_{3}\right] s(x-y)\right) /(9 y u(s-t+u)(s-x+y)) \text {. }
\end{aligned}
$$


The remaining matrix elements can be obtained by the interchange of $p_{1} \leftrightarrow p_{2}, t \leftrightarrow x, u \leftrightarrow y$ from the above results.

$$
\begin{aligned}
& e(5,5) \longleftrightarrow e(1,1) \quad e(6,5) \longleftrightarrow e(2,1) \quad e(6,6) \longleftrightarrow e(2,2) \\
& e(7,5) \longleftrightarrow e(3,1) \quad e(7,6) \longleftrightarrow e(3,2) \quad e(7,7) \longleftrightarrow e(3,3) \\
& e(8,5) \longleftrightarrow e(4,1) \quad e(8,6) \longleftrightarrow e(4,2) \quad e(8,7) \longleftrightarrow e(4,3) \\
& e(8,8) \longleftrightarrow e(4,4) \quad e(6,1) \longleftrightarrow e(5,2) \quad e(7,1) \longleftrightarrow e(5,3) \\
& e(8,1) \longleftrightarrow e(5,4) \quad e(7,2) \longleftrightarrow e(6,3) \quad e(8,2) \longleftrightarrow e(6,4) \\
& e(8,3) \longleftrightarrow e(7,4) .
\end{aligned}
$$




\section{References}

[1] J. Ellis, R. Jaffe; Phys. Rev. D 9 (1974) 1444; ibid. D 10 (1974) 1669.

[2] M. J. Alguard et al. (SLAC); Phys. Rev. Lett. 37 (1978) 1262; ibid. 41 (1978) 70,

J. Ashman et al. (EMC); Phys. Lett. B 206 (1988) 364, Nucl. Phys. B 328 (1989) 1,

B. Adeva et al. (SMC); Phys. Lett. B 302 (1993) 533.

[3] J. Ellis, R. A. Flores, S. Ritz;Phys. Lett. B 198 (1987) 393,

J. Ellis, R. A. Flores;Nucl. Phys. B 307 (1988) 883,

S. J. Brodsky, J. Ellis, M. Karliner;Phys. Lett. B 206 (1988) 309,

M. Glück, E. Reya;Z. Phys. C 39 (1988) 569.

[4] G. Altarelli, G. G. Ross;Phys. Lett. B 212 (1988) 391,

A. V. Efremov, O. V. Teryaev;Dubna Report No. E2-88-287,1988

(published in Czech. Hadron Symposium 1988, p. 302).

[5] G. Altarelli, W. J. Stirling; Particle World 1 (1989) 40,

R. D. Carlitz, J. C. Collins, A. H. Mueller; Phys. Lett. B 214 (1988) 229.

[6] G. Altarelli; Proceedings of the 27th Int. E. Majorana Summer School of Subnuclear Physics, Erice 1989, ed. A. Zichichi, Plenum Press

[CERN-TH. 5675/90], and Proceedings of the HERA-Workshop, Hamburg 1991, eds. W. Buchmüller and G. Ingelmann (DESY, 1992), vol. I, p. 379 [CERN-TH. 6340/91],

G. G. Ross; Proceedings of the 1989 Int. Symposium on Lepton and Photon Interactions at High Energies, ed. M. Riordan (World Scientific, 1990) p. 41,

H. Y. Cheng; Chin. J. Phys. 29 (1991) 67,

E. Reya; Proceedings of the Int. Workshop on High Energy Physics Phenomenology II, Calcutta, 1991, eds. A. Datta, P. Ghose,

A. Raychaudhuri (World Scientific, 1992) [DO-TH 91/09].

[7] Glück, Reya, Vogelsang; Nucl. Phys. B 329 (1990) 347.

[8] G. Baum et al. , Phys. Rev. Lett. 51 (1983) 1135;

see also: V. W. Hughes et al., Phys. Lett. B 212 (1988) 511.

[9] G. Altarelli, in: The Challenging Questions, Proc. 1989 Erice School, ed. A. Zichichi (plenum, New York, 1990).

[10] B. Adeva et. al., Phys. Lett. B 302 (1993) 533. 
[11] P.L. Anthony et al., SLAC preprint SLAC-PUB-6101 (1993).

[12] J. Ellis and M. Karliner, CERN preprint CERN-TH. 6898/93.

[13] Hai-Yang Cheng, C. F. Wai; Phys. Rev. D 46 (1992) 125.

[14] J. G. Körner, E. Mirkes, G.A. Schuler; Int. J. Mod. Phys.A4 (1989) 1781.

[15] T. Brodkorb, J. G. Körner, E. Mirkes, G. A. Schuler; Z. Phys. C-Particles and Fields 44 (1989) 415.

[16] M. Diemoz, F. Ferroni, E. Longo, M. Martinelli Z. Phys. C 39 (1988) 472.

[17] R. K. Ellis, D. A. Ross, A. E. Terrano; Nucl. Phys. B 178 (1981) 421, A. Ali et. al.; Nucl. Phys. B 167 (1980) 454, J.G. Körner et. al.; Nucl. Phys. B 185 (1981) 365 


\section{Figure captions}

Fig. 1 Diagrams for $\gamma^{*} q \rightarrow G q(\mathrm{a})$ and $\gamma^{*} G \rightarrow q \bar{q}(\mathrm{~b})$.

Fig. 2 Diagrams for $\gamma^{*} q \rightarrow q G G(\mathrm{a}), \gamma^{*} G \rightarrow q \bar{q} G$ (b) and $\gamma^{*} q \rightarrow q \bar{q} q$ (c).

Fig. 3 Dependence of the various $O\left(\alpha_{s}\right)$ (a-c) and $O\left(\alpha_{s}^{2}\right)$ (e-f) processes on the total cms energy $\sqrt{s}$.

a,d) parton densities given by set a: eq. (33).

b,e) parton densities given by set b: eq. (34).

$\mathrm{c}, \mathrm{f})$ parton densities given by set c: eq. (35).

The $Q^{2}$ range is restricted to $5 \mathrm{GeV}^{2}<Q^{2}<s$ and the cut-off values in (37) are $y_{c}=0.04$ and $M_{0}=2 \mathrm{GeV}$.

Fig. $4 x$-dependence of the $O\left(\alpha_{s}\right)$ 3-jet processes at $\sqrt{s}=20 \mathrm{GeV}$ for each subprocess and the sum (solid line) of all contributing subprocesses.

a) parton densities given by set a: eq. (33).

b) parton densities given by set b: eq. (34).

c) parton densities given by set c: eq. (35).

The $Q^{2}$ range is restricted to $5 \mathrm{GeV}^{2}<Q^{2}<7 \mathrm{GeV}^{2}$ and the cut-off values in (37) are $y_{c}=0.04$ and $M_{0}=2 \mathrm{GeV}$.

Fig. 5 same as Fig. 4 for $9 \mathrm{GeV}^{2}<Q^{2}<11 \mathrm{GeV}^{2}$.

Fig. $6 W$-dependence of the $O\left(\alpha_{s}\right)$ 3-jet processes at $\sqrt{s}=20 \mathrm{GeV}$ for each subprocess and the sum (solid line) of all contributing subprocesses.

a) parton densities given by set a: eq. (33).

b) parton densities given by set b: eq. (34).

c) parton densities given by set c: eq. (35).

The $Q^{2}$ range is restricted to $5 \mathrm{GeV}^{2}<Q^{2}<7 \mathrm{GeV}^{2}$ and the cut-off values in (37) are $y_{c}=0.04$ and $M_{0}=2 \mathrm{GeV}$.

Fig. 7 same as Fig. 6 for $9 \mathrm{GeV}^{2}<Q^{2}<11 \mathrm{GeV}^{2}$.

Fig. 8 same as fig. 4 for the $O\left(\alpha_{s}^{2}\right) 4$-jet processes.

Fig. 9 same as fig. 6 for the $O\left(\alpha_{s}^{2}\right) 4$-jet processes. 
This figure "fig1-1.png" is available in "png" format from: http://arxiv.org/ps/hep-ph/9403410v1 
This figure "fig2-1.png" is available in "png" format from: http://arxiv.org/ps/hep-ph/9403410v1 
This figure "fig3-1.png" is available in "png" format from: http://arxiv.org/ps/hep-ph/9403410v1 
This figure "fig1-2.png" is available in "png" format from: http://arxiv.org/ps/hep-ph/9403410v1 
This figure "fig2-2.png" is available in "png" format from: http://arxiv.org/ps/hep-ph/9403410v1 
This figure "fig3-2.png" is available in "png" format from: http://arxiv.org/ps/hep-ph/9403410v1 
This figure "fig1-3.png" is available in "png" format from: http://arxiv.org/ps/hep-ph/9403410v1 
This figure "fig2-3.png" is available in "png" format from: http://arxiv.org/ps/hep-ph/9403410v1 
This figure "fig3-3.png" is available in "png" format from: http://arxiv.org/ps/hep-ph/9403410v1 
This figure "fig1-4.png" is available in "png" format from: http://arxiv.org/ps/hep-ph/9403410v1 
This figure "fig2-4.png" is available in "png" format from: http://arxiv.org/ps/hep-ph/9403410v1 
This figure "fig3-4.png" is available in "png" format from: http://arxiv.org/ps/hep-ph/9403410v1 